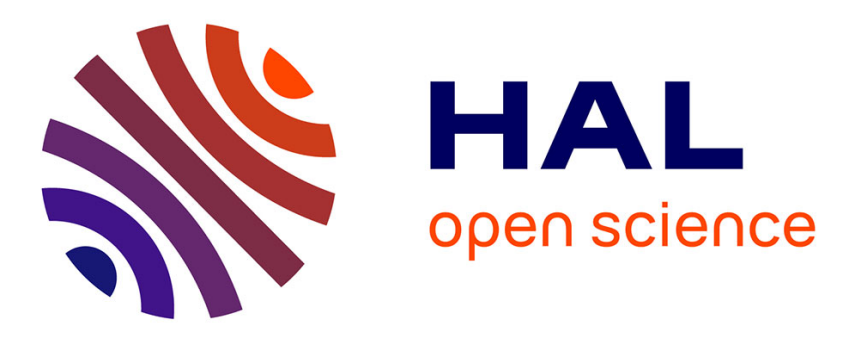

\title{
A Mechanized Semantic Framework for Real-Time Systems
}

\author{
Manuel Garnacho, Jean-Paul Bodeveix, M Filali
}

\section{To cite this version:}

Manuel Garnacho, Jean-Paul Bodeveix, M Filali. A Mechanized Semantic Framework for Real-Time Systems. 11th International Conference on Formal Modeling and Analysis of Timed Systems (FORMATS 2013), Aug 2013, Buenos Aires, Argentina. pp.106-120, 10.1007/978-3-642-40229-6_8 . hal01231769

\section{HAL Id: hal-01231769 \\ https://hal.science/hal-01231769}

Submitted on 20 Nov 2015

HAL is a multi-disciplinary open access archive for the deposit and dissemination of scientific research documents, whether they are published or not. The documents may come from teaching and research institutions in France or abroad, or from public or private research centers.
L'archive ouverte pluridisciplinaire HAL, est destinée au dépôt et à la diffusion de documents scientifiques de niveau recherche, publiés ou non, émanant des établissements d'enseignement et de recherche français ou étrangers, des laboratoires publics ou privés. 


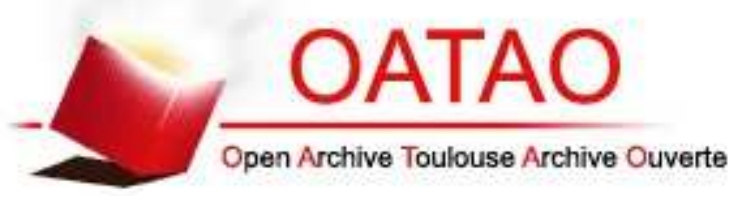

\section{Open Archive TOULOUSE Archive Ouverte (OATAO)}

OATAO is an open access repository that collects the work of Toulouse researchers and makes it freely available over the web where possible.

This is an author-deposited version published in : http://oatao.univ-toulouse.fr/ Eprints ID : 12577

The contribution was presented at FORMATS 2013 :

http://projects.lsv.ens-cachan.fr/formats2013/

Official URL: http://dx.doi.org/10.1007/978-3-642-40229-6_8

To cite this version : Garnacho, Manuel and Bodeveix, Jean-Paul and Filali, Mamoun A Mechanized Semantic Framework for Real-Time Systems. (2013) In: 11th International Conference on Formal Modeling and Analysis of Timed Systems (FORMATS 2013), 29 August 2013 - 31 August 2013 (Buenos Aires, Argentina).

Any correspondance concerning this service should be sent to the repository administrator: staff-oatao@listes-diff.inp-toulouse.fr 


\title{
A Mechanized Semantic Framework for Real-Time Systems *
}

\author{
Manuel Garnacho, Jean-Paul Bodeveix, Mamoun Filali \\ IRIT - CNRS - Université de Toulouse, France
}

\begin{abstract}
Concurrent systems consist of many components which may execute in parallel and are complex to design, to analyze, to verify, and to implement. The complexity increases if the systems have real-time constraints, which are very useful in avionic, spatial and other kind of embedded applications. In this paper we present a logical framework for defining and validating real-time formalisms as well as reasoning methods over them. For this purpose, we have implemented in the CoQ proof assistant well known semantic domains for real-time systems based on labelled transitions systems and timed runs. We experiment our framework by considering the real-time CSP-based language FIACRE, which has been defined as a pivot formalism for modeling languages (AADL, SDL, ...) used in the TOPCASED project. Thus, we define an extension to the formal semantic models mentioned above that facilitates the modeling of fine-grained time constraints of FIACRE. Finally, we implement this extension in our framework and provide a proof method environment to deal with real-time system in order to achieve their formal certification.
\end{abstract}

\section{Introduction}

Real-time concurrent systems consist of many components which may execute in parallel and communicate data or synchronize at a specified time. Therefore, these systems are complicated to design, to analyze, to verify, and finally to implement. The complexity arises from the nondeterminism of behaviors, time constraints and the combinations of ways in which the components can interact. In order to be able to prove or verify properties on such systems one needs to formalize their semantics.

Formal semantics of specification and programming languages are mathematical descriptions of the meaning of programs (and their behaviors) written in these languages. They play a very important role in many areas of computer science such as verification of critical systems because they enable us to formally prove that these systems meet their specifications. Formal semantics of componentbased languages, which are useful to design distributed or concurrent systems, are commonly defined in terms of transition systems. If the language has also realtime features, then its semantics can be expressed as timed transition systems [4] (TTS for short, see Definition 3). For this paper, we address the challenge of

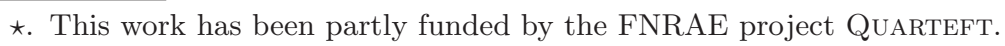


providing a framework that allows to build mechanized reasonings over timed systems within a proof assistant. Doing so requires to mechanize the semantics of timed systems in adapted mathematical terms, which are here theories of transition systems and execution runs (or traces) which include time aspects.

The present work proposes also an extension to the semantic model of transition systems with time constraints proposed by T.Henzinger et al [16] that we call time constrained transition systems (TCTS for short, see Definition 7) since the term TTS is overloaded and already used here. The purpose of this extension is to model more directly and easily some real-time constructs of specification languages and then be able to prove timed temporal properties over critical embedded systems. We define semantic interpretations of systems modeled as TCTS in terms of TTS and timed runs (see Section 2.2) that enable to reason over these models and their time properties. Those semantic interpretations correspond to arrows 2 and 3 of Figure 1 which describes the global architecture of our framework. Arrow 1, which addresses the semantics interpretation of the syntactical constructs of our component-based language (namely FIACRE), goes beyond our current purpose and is not presented here (this is our long term goal).

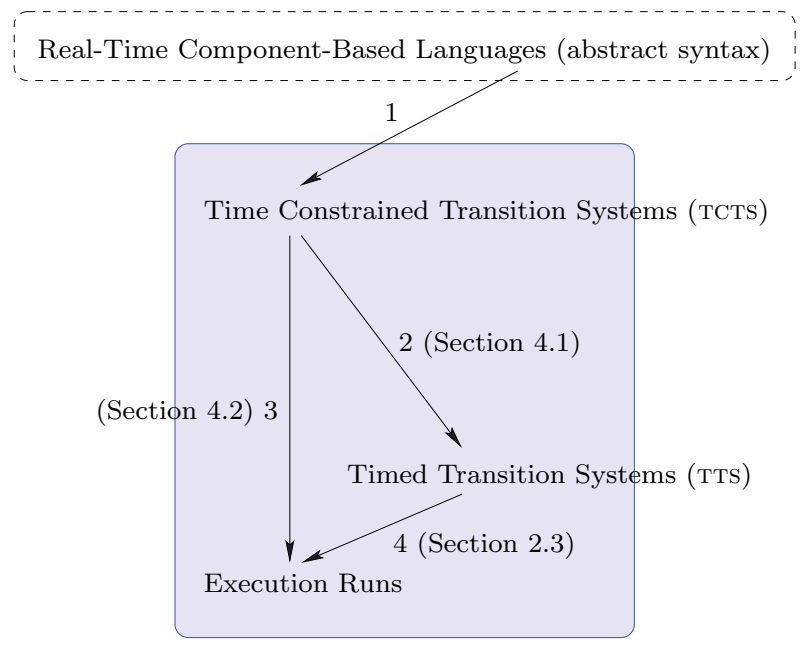

Fig. 1. Schematic description of the architecture of our semantic framework

There are several reasons for wanting to mechanize such theories based on transition systems and timed runs in an interactive theorem-prover rather than building a dedicated automatic tool [13]. The most important reason is certainly that proof assistants like COQ [7], HOL [18] or PVS [22], provide a generic and very expressive proof environment for dealing with problems which cannot be automatically decided. We claim also that the results which are encoded and checked with such tools reach probably the currently highest level of confidence in formal software verification. In other words, proofs built and then checked with, for 
example CoQ, avoid to a very high extent fallacious and incomplete arguments that are so easy and so common to find in standard mathematical proofs. Then, all the proofs that we provide in this paper have been encoded in the calculus of inductive constructions (CIC for short) using the COQ proof assistant, and then automatically checked to guarantee the mathematical soundness of the proofs ${ }^{1}$. However, although all of this formalization work (shaded part of Figure 1) has been carried out in CoQ, we refrained from displaying CoQ syntax here. We adopt standard mathematical notations as much as possible for the sake of clarity and because a large part of this work is about semantics of real-time systems that is widely independent of COQ.

Overview. The remainder of this paper is structured as follows: Section 1.1 introduces the general principles of component-based languages. We describe their timed semantics through a toy example. Section 2 recalls the basis of transition systems and execution runs. Section 3 defines an extension of transition systems with time constraints. Section 4 is dedicated to the semantic interpretations of transition systems in two distinct models that address the dynamic time aspects of real-time systems. Then, in Section 5 we explain why our work has been implemented in the interactive theorem prover CoQ and how it can be used for formal verification. We draw a conclusion and present some directions for future works in Section 6 .

\subsection{Timed Semantics of Component-Based Languages}

Existing specification languages inspired by [17] are useful to represent both behavioral and timing aspects of concurrent (or distributed) real-time systems. For example, FIACRE [6] is defined over two main notions : (1) processes that describe the behavior of sequential components. A process is defined by a set of control states (or positions), each associated with a piece of program built from deterministic constructs available in classical programming languages, nondeterministic constructs (nondeterministic choice of the transition to take, for example), communication events on ports, and jumps to next position; (2) components that describe the composition of processes. A component is defined as a parallel composition of components and/or processes communicating through ports and shared variables.

In this paper we focus only on nondeterministic transitions and timing constraints of synchronizations (composition and priorities are adressed in [12]). FIACRE allows to model real-time behaviors by using timed ports, i.e. ports with their own timer (or clock). A timed transition connects two control states using such a port. Moreover, FIACRE allows to code such transitions in two ways : the first one preserves the clocks of other enabled transitions whereas the second one resets the clock of other enabled transitions.

1. we invite the interested reader to browse the sources of our development at the web address : http://www.irit.fr/ Manuel.Garnacho/Coq/FORMATS13. 
The example presented in Figure 2 describes a process which declares three local ports, $\mathrm{p}, \mathrm{q}$ and $\mathrm{r}$, and two variables of type nat, namely $\mathrm{x}$ and $\mathrm{y}$. In this specification language, local ports are only used to temporize transitions and processes which use those ports do not get "synchronized" with others through those ports. The port $\mathrm{p}$ is time constrained by the interval $[1,3[, \mathrm{q}$ by $] 3, \infty[$ and $r$ by $[1,1]$. Considering $\mathrm{p}$, it means that when a transition which synchronizes on it is enabled, it must fire after waiting at least one (included) and at most three (not included) time units. In the same way, q delays the process P for at least three (not included) time units ${ }^{2}$ and $r$ exactly one unit of time.

Then, we see that in the process $\mathrm{P}$, there are two control states, namely s0 and s1. Only s0 is an initial one, which means that the process has to start its execution from it. But let us first take a look at s1 : from this control state, a nondeterministic choice is defined with the construction select. This choice includes two concurrent transitions : the first one that synchronizes on $p$ then adds to $y$ the value of $x$; the second one that synchronizes on $q$ then assigns to $\mathrm{x}$ the value of $\mathrm{y}$ plus 2. Afterward, each of those transitions jumps to a next control state with the construction to, i.e. it jumps back to $\mathrm{s} 1$ in the case of an action on $\mathrm{p}$ and goes to $\mathrm{s} 0$ in the other case. The timed-semantics of the construction to, consists in resetting the timer of all transitions included in the same nondeterministic choice (we provide in comments the set of ports that have to be reset for each transition). This notion will be formalized in Section 3, thanks to an extension of the semantic model introduced in [16]. Consequently, from the control state $\mathrm{s} 1$ the process $\mathrm{P}$ is never synchronized on $\mathrm{q}$, so the variable $\mathrm{x}$ will never be assigned and it never goes back to s0. Indeed, after every single unit of time the process will be synchronized on $\mathrm{p}$ before strictly two more time units, after which it resets the timer of $\mathrm{q}$ before having waited enough time to synchronize on it. Thus, the second transition of the nondeterministic choice from $\mathrm{s} 1$ of $\mathrm{P}$ is dead code due to the time constraints of $\mathrm{P}$.

Now, through the same example we present another construction that loops to the current control state but with a different timed-semantics. This construction is still related to the nondeterministic choice of transitions and is defined with the keyword loop. It consists in going back to the current control state while preserving the time already waited by other transitions (which were not taken) included in the same nondeterministic choice as the one that has been taken. The clock of the taken transition is reset however. For example, in the nondeterministic choice from the control state $\mathrm{s} 0$ of $\mathrm{P}$, the first transition loops after synchronizing on $r$ and then increments $\mathrm{x}$. This transition occurs after exactly every single time unit and then only resets its own timer. On the other hand, the nondeterministic choice allows the process to synchronize on $\mathrm{q}$ at any time after being continuously enabled for at least three units of time. Note that the transition through q from s0 may never occur because of its unconstrained maximal delay. Proving such semantics properties on the value of $\mathrm{x}$ and $\mathrm{y}$ or that the transition through $\mathrm{q}$ from $\mathrm{s} 1$ is dead code, can be as much crucial as difficult.

2. Time bounds are either integral or infinite. Elapsing of time is continuous. 


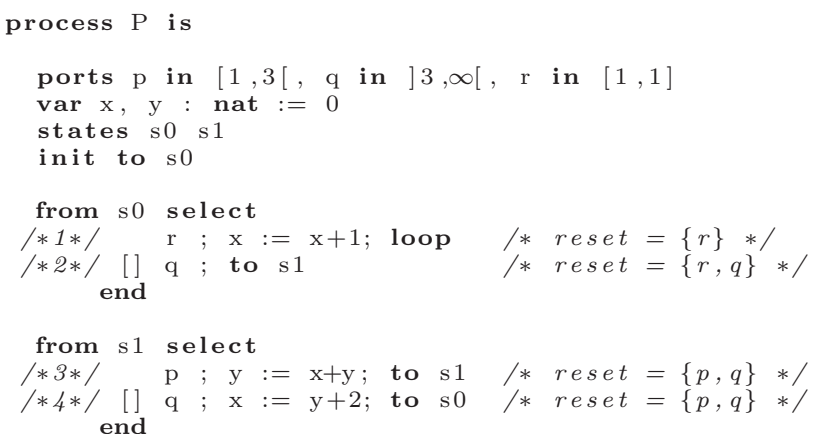

Fig. 2. An example real-time process specified in FIACRE

Related Work. Starting with the work of T. Henzinger et al. [16,15], we extend their mathematical semantic model for specifying real-time processes such as the one presented in Figure 2. All definitions and theorems presented in the following are directly derived from a mechanization developed in CoQ. This part of our contributions connects to the previous work of R. Cardell-Oliver et al. [14] about the embedding in HOL of [16] and C. Paulin-Mohring about the formalization of timed automata in CoQ [20]. With respect to the Petri Net community, our work is in the spirit of B. Berard et al. [4]. However, we are concerned by a component-based language with states and ports and we go further since we adress its mechanization. Morever, our work is not in the same scope as [13] because our goal is not to develop an automatic verification tool certified in CoQ. Our purpose is to provide a real-time semantic framework which can be used to formalize real-time patterns and more generally real-time specification languages. As a matter of fact, we present in the following the formalization of the FIACRE language where we are especially interested in the semantic differences between the contructs loop and to (see Section 1.1). Also, the proof environment of our framework allows to certify transformations between real-time formalisms. For instance in [8], we are concerned by the transformation from AADL to FIACRE, which requires formal certification for avionic or spatial applications.

\section{Semantics Kernel}

This section is dedicated to the basis of classical semantic notions we use in our work. These notions are of two kinds: timed transition systems and timed execution runs [4]. Moreover, both include time and since time is dense in most real-time specification languages (including FIACRE), we formalize time in this paper with non-negative real numbers : $\mathbb{R}^{+}$. However, in our CoQ mechanization we have developed an axiomatic theory of time ${ }^{3}$ which can be instantiated by constructive reals [13] as well as by nat to encode discrete time (if necessary).

3. http://www. irit.fr/ Manuel. Garnacho/Coq/FORMATS13/time.html. 
These semantic models enable us to describe formally the dynamics of real-time systems, mixing the evolution of their states and the progress of time, to reason over those with rigorous logical methods.

\subsection{Transition Systems}

First of all, we remind the basic model of labelled transition systems [2] that is usually used to give a mathematical representation of computer programs.

Definition 1 (Labelled Transition Systems). A labelled transition system (LTS for short) is a 4-tuple lts $\stackrel{\text { def }}{=}\langle L, S$, init, next $\rangle$, where : $L$ is the set of labels of lts; $S$ is a set of states (or stores); init is a non-empty subset of $S$ that defines the initial states of lts; and next defines the set of transitions of lts that are triplets of the form $\left(\right.$ sto, $\ell$, sto $\left.^{\prime}\right) \in S \times L \times S$, also denoted as sto $\stackrel{\ell}{\longrightarrow}$ sto', where sto $\in S$ is the source state, $\ell \in L$ is the label and sto' $\in S$ the target state of the transition.

Definition 2. Assuming a LTS, lts $\stackrel{\text { def }}{=}\langle L, S$, init, next $\rangle$, a label $\ell \in L$ is enabled from a state sto $\in S$, if there exists a state sto' $\in S$ such that the triplet (sto, $\ell$, sto' $^{\prime}$ ) belongs to next. Formally, we define the predicate enb over $S \times L$ as $\boldsymbol{e n b}_{\text {sto }}(\ell) \stackrel{\text { def }}{=} \exists s t o^{\prime} \in S, \boldsymbol{n e x t}($ sto, $\ell$, sto' $)$

Definition 3 (Timed Transition Systems). A timed transition system (TTS for short) over a set of labels $L$, is a LTS over $L \cup \mathbb{R}^{+},\left\langle L \cup \mathbb{R}^{+}, S\right.$, init, $\boldsymbol{t}_{-}$next $\rangle$, that satisfies the following properties:

- zero delay : $\forall$ sto $\in S$, sto $\stackrel{0}{\longrightarrow}$ sto

- time-determinism : $\forall$ sto, sto', sto" $\in S, \forall \delta \in \mathbb{R}^{+}$,

- additivity : $\forall$ sto, sto', sto' $\in S, \forall \delta, \delta^{\prime} \in \mathbb{R}^{+}$,

$$
\text { sto } \stackrel{\delta}{\longrightarrow} \text { sto } \Rightarrow \text { sto } \stackrel{\delta}{\longrightarrow} \text { sto" } \Rightarrow \text { sto' }=\text { sto }{ }^{\prime \prime}
$$

$$
\text { sto } \stackrel{\delta}{\longrightarrow} \text { sto } \Rightarrow \text { sto } \stackrel{\delta^{\prime}}{\longrightarrow} \text { sto } \Rightarrow \text { sto } \stackrel{\delta+\delta^{\prime}}{\longrightarrow} \text { sto }
$$

- continuity $: \forall$ sto, sto" $\in S, \forall \delta^{\prime}, \delta^{\prime \prime} \in \mathbb{R}^{+}$,

$$
\text { sto } \stackrel{\delta^{\prime}+\delta^{\prime \prime}}{\longrightarrow} \text { sto } \Rightarrow \exists \text { sto }, \text { sto } \stackrel{\delta^{\prime}}{\longrightarrow} \text { sto } \wedge \text { sto } \stackrel{\delta^{\prime \prime}}{\longrightarrow} \text { sto }^{\prime \prime}
$$

\subsection{Executions}

We remind now the classical representation of runs in a timed context, that is useful to represent the behaviors of systems and then reason with temporal linear logic [21] about those.

Definition 4 (Timed execution states). With respect to a set of states $S$ and a set of events L, a timed execution state (TES for short), s, is a triplet of the form 〈sto, evt, now〉 where :

- sto $_{s} \in S$, is the state (or store) reached in $s$

- evt $\in L \cup\{$ start $\} \cup\{d i v\}$, is the event that has reached $s$.

- now n $^{+}$gives the current global time (or date) in $s$. 
Remarks. The two special events start and div are seen, respectively, at the beginning of a run and whenever time is diverging (in case of deadlock, for instance) in a run.

Definition 5 (Runs). A run (or execution sequence) is a function $\sigma$, from $\mathbb{N}$ to TES, that represents a (possibly finite) sequence of events all of which reach a state at a given date. For a TES $s$ reached at a position $i$ of $\sigma$, we have $\sigma_{i}=s$.

Remarks. For the sake of conciseness, we allow ourselves to substitute $s\left(\sigma_{i}\right)$ by $i$ in the following whenever it might simplify the notations (as below). Also, according to our representation of runs, finite ones are encoded using a repetition of the special event div after the last state.

Definition 6 (Well formed runs). According to the previous definition, a run $\sigma$ is well formed if it satisfies the following properties : start : evt $t_{0}=$ start $\wedge$ now $_{0}=\delta_{\sigma}^{0}$; and monotonicity $: \forall i \in \mathbb{N}$, now now $_{i+1}$, where $\delta_{\sigma}^{0}$ is the initial delay associated to the beginning (the event start) of the run $\sigma$.

Remark. This definition of timed runs does not satisfy the progress property $\left(\forall r \in \mathbb{R}^{+}, \exists i \in \mathbb{N}, r \leq\right.$ now $\left._{i}\right)[16]$ because of Zeno behaviors (that include an infinite number of discrete steps in a finite amount of time) that may be generated from the component-based source language, as for instance in FIACRE, that we want to capture.

\subsection{Semantics of Timed Transition Systems in terms of Runs}

We define now the semantics of TTS in terms of runs (arrow 4 of Figure 1).

Predicates. In order to describe the semantics of TTS $s$ in terms of runs, we have to define two predicates that express when a given label may be taken and when a label is taken. Assume a given run $\sigma$ at some position $i, \sigma-e \boldsymbol{n} \boldsymbol{b}_{i}(\ell)$ means that the label $\ell$ may be taken at $i$ and $\operatorname{taken}_{i}(\ell)$ means that $\ell$ is taken at $i$. Formally: - $\sigma$-enb $\boldsymbol{e n}_{i}(\ell) \stackrel{\text { def }}{=} \exists$ sto, $_{\text {sto }} \in S, \exists \delta \in \mathbb{R}^{+}, \boldsymbol{n e x t}\left(\right.$ sto $_{i}, \ell$, sto $) \wedge \boldsymbol{n e x t}($ sto, $\delta$, sto' $)$

- $\operatorname{taken}_{i}(\ell) \stackrel{\text { def }}{=} \operatorname{evt}_{i+1}=\ell$ (sto $_{i+1}$ corresponds to one of the sto' above)

Remark. The intermediate state, sto, used in the definition of $\sigma-\boldsymbol{e n} \boldsymbol{b}$ is needed because the runs that we consider do not include explicit duration. There are other formalisms of execution runs where time passing is explicit (mixing durations and events) but here, to avoid Zeno phenomena (coming from the TTS $s$ and not from the systems theirselves) in runs, we only consider events.

Semantic Interpretation in terms of Runs. The run-semantics of a TTS, $t t s \stackrel{\text { def }}{=}\left\langle L \cup \mathbb{R}^{+}, S\right.$, init, $\left.\boldsymbol{t}_{-} \boldsymbol{n e x \boldsymbol { t }}\right\rangle$, is a set of well formed runs $\Sigma(t t s)$, that expresses all possible behaviors of $t$ ts at execution, such as each of those has to fulfill that init $\left(\right.$ sto $\left._{0}\right)$ and the following step condition : 
$\forall i$, either

$\exists \ell$ such that $\exists$ sto $\in S, \exists \delta \in \mathbb{R}^{+}, \boldsymbol{t} \_\boldsymbol{n e x t}\left(\right.$ sto $_{i}, \ell$, sto $) \wedge \boldsymbol{t} \_\boldsymbol{n e x t}\left(\right.$ sto, $\delta$, sto $_{i+1}$ ) and now $_{i+1}=$ now $_{i}+\delta$ and $\operatorname{taken}_{i}(\ell)$ (a label is taken)

or $\neg \exists \ell$ such that $\sigma-\boldsymbol{e n} \boldsymbol{b}_{i}(\ell)$ and $\forall j>i$, sto ${ }_{j}=$ sto $_{i} \wedge \mathrm{evt}_{j}=\operatorname{div}$ (time is diverging).

Graphically, when a label $\ell$ is taken at some position $i$, it can be represented as $\sigma_{i} \stackrel{\ell}{\longrightarrow} s \stackrel{\delta}{\longrightarrow} \sigma_{i+1}$, where $s$ is an implicit intermediate TES which is not visible in the run, defined as $\left\langle\right.$ sto $_{i+1}$, evt $_{i+1}$, now $\left._{i}\right\rangle$.

\section{A Time Constrained Model for Real-Time Systems}

We give in this section an extended definition of the semantics model proposed by T. Henzinger et al. in [16] that deals with advanced (i.e. fine-grained) timed constructs such as the ones presented in Section 1.1. As in [16], we incorporate real-time constraints to LTS in order to ensure that a labelled transition is fired neither too early nor too late. An extension to their initial model is the so-called reset relation that specifies which clocks (or timers) are reset after the firing of a given transition. We call this model Time Constrained Transition Systems (TCTS for short) since time features are only expressed as syntactic timed constraints on transitions. We have introduced this semantics model in order to verify realtime processes such as the one presented in Figure 2 more easily. Moreover, the exhibited semantic model is interesting by itself since it can be reused to define the semantics of real-time component-based langages such as FIACRE [11] or BIP[3] or even high-level specification models such as Timed Petri Nets with read-arcs [4]. We also present two timed-semantic interpretations, corresponding to arrows 2 and 3 of Figure 1.

\subsection{Time Constrained Transition Systems}

In order to reason about timing of transitions, we identify each transition by its name. For instance, according to the process P of Figure 2, its corresponding TCTS include four names, one for each of the four possible transitions from its two nondeterministic choices, even if the number 2 and 4 synchronize both on q. Moreover, we suppose that there is an implicit clock (while time is explicit in TTS) associated to every transition. Then, this model allows to specify static time constraints over the transitions of a given system.

Definition 7. A Time Constrained Transition System, namely tcts, is a 8-tuple $\langle L, T, S, I b /$, init, next, $\mathcal{R}, I\rangle$, where $\langle L, S$, init, next $\rangle$ is a LTS, and :

- $T$ is the set of (the names of) transitions of tcts.

- Ibl is a function from $T$ to $L$, that associates to every transition a label.

- $\mathcal{R}$ is the reset transition relation. $\left(t r, t r^{\prime}\right) \in \mathcal{R}$ states that at execution, the firing of tr resets the implicit clock of $t^{\prime}$. Otherwise, the implicit clock associated to $t^{\prime}$ keeps running. For all $t r \in T,(t r, t r) \in \mathcal{R}$ ( $\mathcal{R}$ is refelexive). 
- I is a function that assigns to every label $\ell \in L$ a non-empty interval of $\mathbb{R}^{+}$. $I_{\ell}($ or $I(\ell))$ specifies both minimal (lower) and maximal delay (upper bound) to elapse once $\ell$ has been enabled (see Definition 2).

We introduce the downward and the upward closure of an interval $I_{\ell}$ defined respectively by $\overleftarrow{I_{\ell}} \stackrel{\text { def }}{=}\left\{t \mid \exists r \in I_{\ell}, t \leq r\right\}$ and $\vec{I}_{\ell} \stackrel{\text { def }}{=}\left\{t \mid \exists r \in I_{\ell}, r \leq t\right\}$. For example if $I_{\ell}$ is defined by the interval $[\min , \max ]$ then $\overleftarrow{I}_{\ell}$ is $[0, \max ]$ and $\vec{I}_{\ell}$ is $[\min , \infty[$.

Example 1. Consider the process given in Figure 2, we define its corresponding TCTS as $\langle L, T, S$, Ibl, init, next, $\mathcal{R}, I\rangle$, where :

- L $\stackrel{\text { def }}{=}\left\{\ell_{p}, \ell_{q}, \ell_{r}\right\}$

- $S \stackrel{\text { def }}{=}\left\{(x, y\right.$, loc $) \mid x, y \in \mathbb{N} \wedge$ loc $\left.\in\left\{l_{0}, l_{1}\right\}\right\}$

- $\forall$ sto $\in S, \boldsymbol{i n i t}($ sto $) \stackrel{\text { def }}{=}$ sto $(x)=0 \wedge \operatorname{sto}(y)=0 \wedge$ sto $(l o c)=l_{0}$

- $\operatorname{next}\left(\right.$ sto, $\ell$, sto $\left.^{\prime}\right) \stackrel{\text { def }}{=}$

$$
\begin{aligned}
& \wedge\left(\begin{array}{l}
\text { sto }(l o c)=l_{0} \Rightarrow \bigvee\left(\begin{array}{l}
\ell=\ell_{r} \wedge \text { sto }^{\prime}=\operatorname{sto}[\operatorname{sto}(x)+1 / x] \\
\ell=\ell_{q} \wedge \text { sto }^{\prime}=\operatorname{sto}\left[l_{1} / l o c\right]
\end{array}\right) \\
\operatorname{sto}(l o c)=l_{1} \Rightarrow \bigvee\left(\begin{array}{l}
\ell=\ell_{p} \wedge \operatorname{sto}^{\prime}=\operatorname{sto}[\operatorname{sto}(x)+\operatorname{sto}(y) / y] \\
\ell=\ell_{q} \wedge \operatorname{sto}^{\prime}=\operatorname{sto}[\operatorname{sto}(y)+2 / x]\left[l_{0} / l o c\right]
\end{array}\right)
\end{array}\right) \\
& -T \stackrel{\text { def }}{=}\left\{t r_{1}, t r_{2}, t r_{3}, t r_{4}\right\} \\
& \text { - }\left|\mathrm{bl}\left(t r_{1}\right) \stackrel{\text { def }}{=} \ell_{r}\right| \mathrm{|b|}\left(t r_{2}\right) \stackrel{\text { def }}{=} \ell_{q}\left|\operatorname{|b|}\left(t r_{3}\right) \stackrel{\text { def }}{=} \ell_{p}\right| \mathrm{Ib|}\left(t r_{4}\right) \stackrel{\text { def }}{=} \ell_{q} \\
& -\mathcal{R} \stackrel{\text { def }}{=}\left\{\left(t r_{i}, t r_{i}\right) \mid 1 \leq i \leq 4\right\} \cup\left\{\left(t r_{2}, t r_{1}\right),\left(t r_{3}, t r_{4}\right),\left(t r_{4}, t r_{3}\right)\right\} \\
& \text { - } I_{\ell_{p}} \stackrel{\text { def }}{=}\left[1,3\left[, I_{\ell_{q}} \stackrel{\text { def }}{=}\right] 3, \infty\left[\text { and } I_{\ell_{r}} \stackrel{\text { def }}{=}[1,1]\right. \text {. }\right.
\end{aligned}
$$

Remarks. Because of the functional relation from $T$ to $L$, we consider (by abuse of notation) in the remaining that, for any TCTS, next is also defined over $S \times$ $T \times S$. Furthermore, assuming a TCTS, tcts $\stackrel{\text { def }}{=}\langle L, T, S$, Ibl, init, next, $\mathcal{R}, I\rangle$, a transition $\operatorname{tr} \in T$ is enabled from a state sto $\in S$, if there is a state sto' $\in S$ such that the triplet (sto, $t r$, sto') belongs to next. Formally, we extend the predicate

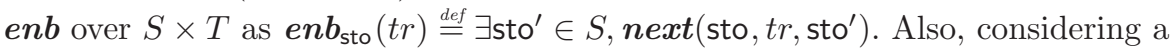
transition $t r \in T$, we write $I_{t r}$ instead of $I_{\mathrm{|b|}(t r)}$ in order to simplify notations.

As we have seen, TCTS is a mathematical model that allows to specify time aspects of real-time systems. We have also defined the composition of TCTS to model concurrent or distributed aspects of systems in the same formalism (see [12]). The semantics of this composition is based on the composition of LTS. However, due to lack of space, we choose to focus on the real-time aspects here.

\section{Semantic Interpretations}

We consider in this section two models to interpret TCTS w.r.t. time semantic aspects. The first one is TTS with explicit time steps together with time assumptions (see Definition 3). This model allows to reason over behaviors of a given TCTS using a branching time logic as [10] or (bi-)simulation relations [4]. 
Secondly, TCTs timing aspects are also interpreted as sets of runs, that describe the executions of a TCTS as sequences of timed events. This model allows to define and to prove the satisfaction of temporal properties expressed in a linear temporal logic [21] by a given TCTS.

\subsection{Semantics of TCTSs in terms of TTSs}

Now, we want to give the semantics of TCTS in terms of TTS, interpreting time constraints by timed transitions. The purpose, among others, is to be able to reason on TCTS $s$ at the TTS level.

So, given a TCTS, tcts $\stackrel{\text { def }}{=}\langle L, T, S$, Ibl, init, next, $\mathcal{R}, I\rangle$, we define a corresponding TTS through the semantics function $\llbracket \rrbracket:$ TCTS $\rightarrow$ TTS, such that $\llbracket$ tcts $\rrbracket=\langle L \cup$ $\mathbb{R}^{+}, \mathcal{S}, \boldsymbol{i n i t}, \boldsymbol{t} \_$next $\rangle \in \mathrm{TTS}$, where :

- a state of $s \in \mathcal{S}$ is a pair over $S \times\left(T \rightarrow \mathbb{R}^{+}\right)$, such that $s \stackrel{\text { def }}{=}\langle$ sto, clk $\rangle$ with :

$\diamond$ sto $_{s} \in S$ is a store of the underlying TCTS.

$\diamond \mathrm{clk}_{s}: T \rightarrow \mathbb{R}^{+}$associates to every transition $t r \in T$ an explicit clock that is needed to count elapsed time since $t r$ is enabled.

$\diamond$ and for every $t r \in T$, if $\boldsymbol{e n} \boldsymbol{b}_{\text {sto }_{s}}(t r)$ then $\mathrm{clk}_{s}(t r) \in \overleftarrow{I_{t r}}$

- $\boldsymbol{t}_{-} \boldsymbol{n} \boldsymbol{e x \boldsymbol { t }}$ is a predicate over $\mathcal{S} \times\left(L \cup \mathbb{R}^{+}\right) \times \mathcal{S}$ defined as :

$\forall s \in \mathcal{S}, \forall s^{\prime} \in \mathcal{S}, \forall \ell \in L \cup \mathbb{R}^{+}, \bigwedge\left(\begin{array}{c}\ell \in L \Rightarrow \operatorname{discrete}\left(s, \ell, s^{\prime}\right) \\ \ell \in \mathbb{R}^{+} \Rightarrow \operatorname{delay}\left(s, \ell, s^{\prime}\right)\end{array}\right)$, with :

$\diamond \forall s \in \mathcal{S}, \forall s^{\prime} \in \mathcal{S}, \forall \ell \in L$, discrete $\left(s, \ell, s^{\prime}\right) \stackrel{\text { def }}{=} \exists \operatorname{tr} \in T, \mathrm{lbl}(t r)=\ell$ and

$$
\begin{aligned}
& \operatorname{next}\left(\mathrm{sto}_{s}, t r, \text { sto }_{s^{\prime}}\right) \text { and } \mathrm{clk}_{s}(t r) \in \overrightarrow{I_{t r}} \\
& \text { and } \forall t r^{\prime}, \mathrm{clk}_{s^{\prime}}\left(t r^{\prime}\right)=\left\{\begin{array}{l}
0 \text { if } \neg e n b_{\text {sto }_{s}}\left(t r^{\prime}\right) \vee\left(t r, t r^{\prime}\right) \in \mathcal{R} \\
\mathrm{clk}_{s}\left(t r^{\prime}\right) \text { else }
\end{array}\right.
\end{aligned}
$$

In other words, considering two timed states $s$ and $s^{\prime}$ of $\llbracket t c t s \rrbracket$, the label of a discrete transition $t r$ links them if (1) $t r$ is a transition between sto $s$ and sto $_{s^{\prime}}$ in the source TCTS; (2) since enabled, $t r$ is delayed enough time units (according to the specification $I_{t r}$ ); and (3) for every other enabled transition $t r^{\prime}$, its clock is preserved if and only if $t r$ does not reset it (clocks are set to 0 for all other transitions, $t r$ included since $(t r, t r) \in \mathcal{R})$.

$\diamond \forall s \in \mathcal{S}, \forall s^{\prime} \in \mathcal{S}, \forall \delta \in \mathbb{R}^{+}, \operatorname{delay}\left(s, \delta, s^{\prime}\right) \stackrel{\text { def }}{=} \forall t r \in T$,

$\mathrm{clk}_{s^{\prime}}(t r)=\mathrm{clk}_{s}(t r)+\delta$

and if $\boldsymbol{e n} \boldsymbol{b}_{\text {sto }_{s}}(t r)$ then we must have $\mathrm{clk}_{s}(t r)+\delta \in \overleftarrow{I_{t r}}$ and sto $_{s^{\prime}}=$ sto $_{s}$

In other words, considering two timed states $s$ and $s^{\prime}$ of $\llbracket t c t s \rrbracket$, time passes ( $\delta$ units) between them if for all transitions $t r$ of tcts, (1) its clock goes $\delta$ time units from $s$ to $s^{\prime}(2)$ after being enabled $\delta$ more units time, the maximal delay to take $t r$ is not reached; and (3) stores are the same.

Theorem 1. $\forall t c t s:$ TCTS, tcts $\rrbracket:$ TTS. 
The proof consists in proving for any tcts $\in$ TCTS, that its semantic interpretation, $\llbracket t c t s \rrbracket$, satisfies the four properties of TTSs (see Definition 3).

\subsection{Semantics of TCTSs in terms of Timed Runs}

The behavior of a TCTS can be expressed by a set of runs (see Definition 5) in order to reason about real-time systems using temporal logics. Here, according to the definition of TCTS with the set $\mathcal{R}$, we need to extend TES $s$ of runs in order to deal with resetting of clocks. Runs of a TCTS are now sequences of TES $s$ as in Definition 8 , but with an additional predicate on the names of transitions.

Definition 8. Assuming a TCTS, tcts $\stackrel{\text { def }}{=}\langle L, T, S,| \boldsymbol{b} /$, init, $\boldsymbol{n e x t}, \mathcal{R}, I\rangle$, a timed execution state of tcts, $s$, is now a 4-tuple 〈sto, evt, now, $\sigma$-reset $\rangle$ where :

- sto $_{s} \in S$, is the state (or store) reached in $s$

- evt $t_{s} \in L \cup\{$ start $\} \cup\{d i v\}$, is the event by which $s$ is attained.

- now $\in \mathbb{R}^{+}$gives the global time in $s$.

- $\sigma$-reset s $_{s}$ a function from $T$ to $\mathbb{B}$ (or a predicate over $T$ ) that indicates which transitions have had their implicit clock reset when s has been reached.

Predicates. Assume a given run $\sigma$ at some position $i, \sigma-\boldsymbol{e n b}_{i}(t r)$ means that the transition $\operatorname{tr} \in T$ may be taken at $i$ and taken $_{i}(t r)$ means that $t r$ is taken at $i$. Formally :

- $\sigma-\boldsymbol{e n b _ { i }}(t r) \stackrel{\text { def }}{=} \exists$ sto $\in S, \boldsymbol{n e x t}\left(\right.$ sto $_{i}, t r$, sto $)$

- taken $_{i}(t r) \stackrel{\text { def }}{=} \bigwedge\left(\begin{array}{l}\operatorname{evt}_{i+1}=\mathrm{lbl}(t r) \\ \boldsymbol{n e x t}_{\left(\text {sto }_{i}, t r, \text { sto }_{i+1}\right)} \\ \forall t r^{\prime}, \sigma-\text { reset }_{i+1}\left(t r^{\prime}\right) \Leftrightarrow\left(t r, t r^{\prime}\right) \in \mathcal{R}\end{array}\right)$

Remark. Here, the predicate taken() is more sophisticated than in Section 2.3 because it deals with $T$ and no more with $L$. Indeed, the first condition of the conjunction above is not enough because two transitions of $T$ might satisfy it.

Run-Semantics of a TCTS. Now, we are able to express the semantic interpretation of a TCTS in terms of runs, as we did in Section 2.3 with TTS. Consider a TCTS, tcts $\stackrel{\text { def }}{=}\langle L, T, S$, Ibl, init, next, $\mathcal{R}, I\rangle$, we define below the set of well formed runs that we call $\Sigma(t c t s)$. Every run $\sigma$ of $\Sigma(t c t s)$, which expresses a behavior of tcts, has to fulfill the four following conditions :

Initial timed execution state condition : $\boldsymbol{i n i t}\left(\mathrm{sto}_{0}\right) \wedge$ now $_{0} \in \overrightarrow{I_{\mathrm{evt}_{1}}}$

In other words, any run must start from an initial state of its underlying TCTS and the taken transition at position 0 has been delayed enough.

Next timed execution state condition :

$\forall i \in \mathbb{N}$, either $\exists t r \in T$ such that $\operatorname{taken}_{i}(t r)$ (a discrete transition is taken) or $\neg \exists t r \in T$ such that $\sigma-\boldsymbol{e n} \boldsymbol{b}_{i}(t r)$ and sto $_{i}=$ sto $_{i+1}=$ sto $_{i+2}=\ldots \wedge$ 


$$
\sigma_{i} \stackrel{d i v}{\longrightarrow} \sigma_{i+1} \stackrel{d i v}{\longrightarrow} \ldots \text { (time diverging) }
$$

In other words, a next timed execution state can be reached from a state at position $i$ of $\sigma$ if either a discrete transition can be taken or a deadlock is met and time goes to infinity (the tag event div was introduced especially for this purpose in order to distinguish deadlock from the identity transition).

Lower bound condition : $\forall t r \in T$, and $\forall j \in \mathbb{N}$, if $\boldsymbol{t a k e n}_{j}(t r)$ then

$$
\begin{aligned}
& \text { now }_{j} \in \overrightarrow{I_{t r}} \text { and } \\
& \forall i \in \mathbb{N} \text { such that } i<j, \\
& \quad \text { if } \text { now }_{j}-\text { now }_{i} \notin \overrightarrow{I_{t r}} \text { then } \sigma-\text { enb }_{i}(t r) \wedge \neg \sigma-\text { reset }_{i+1}(t r)
\end{aligned}
$$

In other words, a transition $t r$ can be taken only after being continuously enabled for enough (means that it is delayed for at least the lower bound of $I_{t r}$ ) time units without being reset by the execution of another transition. The condition now $_{j} \in \overrightarrow{I_{t r}}$ is required in the case where $t r$ is enabled since the start of the execution. Indeed, in this case the premise now $_{j}-$ now $_{i} \notin \overrightarrow{I_{t r}}$ is useless and we need to know that $t r$ has been continuously enabled even before the position $\sigma_{0}$. It seems that this case has been omitted in [16].

Upper bound condition : $\forall t r \in T$ and $\forall i \in \mathbb{N}$,

$$
\begin{aligned}
& \exists j \in \mathbb{N} \text { such that } i \leq j \text { and } \text { now }_{j}-\text { now }_{i} \in \overleftarrow{I_{t r}} \\
& \text { and if } \sigma \text {-enb } \\
& j
\end{aligned}
$$

In other words, once enabled, a transition $t r$ is not delayed for too long or it has been reset meanwhile. A transition cannot be continuously enabled for more than the upper bound of $I_{t r}$ without being taken or reset.

\subsection{Soundness of the Semantic Interpretations}

Now we want to establish the soundness of our semantic interpretations by proving that the runs built straight from a given TCTS are the same that those built from its semantic interpretation in terms of a TTS. To do so, we must first define a semantic interpretation from timed execution state associated to TCTS $s$ to the ones associated to their corresponding TTSs. So, we introduce the function $\gamma$ from $\Sigma(t c t s)$ to $\Sigma(\llbracket t c t s \rrbracket)$, where states are in $\mathcal{S} \stackrel{\text { def }}{=} S \times\left(T \rightarrow \mathbb{R}^{+}\right.$) (see Section 4.1), i.e. every state associates a clock to every transition.

Definition 9. $\forall t c t s:$ TCTS $, \forall \sigma \in \Sigma(t c t s), \forall i \in \mathbb{N}, \gamma\left(\sigma_{i}\right) \stackrel{\text { def }}{=}$ $\left\{\begin{array}{l}\left\langle\left\langle\text { sto }_{0},\left(\lambda t r \in T . \delta_{\sigma}^{0}\right)\right\rangle, \text { start }, \text { now }_{0}\right\rangle \text { if } i=0, \\ \left.\left.\left\langle\begin{array}{l}\text { sto }_{i}, \\ \lambda t r \in T .\end{array}, \begin{array}{l}\delta \text { if } \neg \sigma-\text { enb }_{i-1}(\text { tr }) \text { or } \sigma-\text { reset }_{i}(t r) \\ \text { clk }_{\gamma\left(\sigma_{i-1}\right)}(t r)+\delta \text { otherwise }\end{array}\right)\right\rangle, \text { evt }_{i}, \text { now }_{i}\right\rangle \text { else }\end{array}\right.$ where $\delta \stackrel{\text { def }}{=}\left(\right.$ now $_{i}-$ now $\left._{i-1}\right)$ and $\delta_{\sigma}^{0}$ is the initial delay of $\sigma$, which is now . . 
Then, we define the semantics interpretation of runs of TCTSs in terms of runs of corresponding TTSs as $\forall$ tcts $\in \mathrm{TCTS}, \forall \sigma \in \Sigma(t c t s), \gamma(\sigma) \stackrel{\text { def }}{=} \forall i \in \mathbb{N}, \gamma\left(\sigma_{i}\right)$.

Theorem 2. $\forall t c t s:$ TCTS, $\forall \sigma: \mathbb{N} \rightarrow \mathrm{TES}, \sigma \in \Sigma(t c t s) \Leftrightarrow \gamma(\sigma) \in \Sigma(\llbracket t c t s \rrbracket)$.

\section{Mechanizing and Reasoning with a Proof Assistant}

We explain in this section why we have implemented during the last two years the formalization work presented in this paper in an interactive theorem prover based on type-theory. First of all, the purpose is to validate with a very high level of confidence all the theorems about semantic interpretations of TCTS that we have presented previously. Moreover, using formal fundational proofs can also be useful to go beyond model-checking techniques and capabilities [13], when it is needed for example to check parameterized systems. To make that practical, we have deeply embedded in CoQ a timed linear temporal logic ${ }^{4}$ (called timedSELTL) which is an extension of the State/Event linear temporal logic (SE-LTL) [9] since it embeds time intervals within temporal operators, together with a proof methodology dedicated to the formal certification of real-time systems.

Definition 10. A timed-SELTL formula $f$ is defined inductively as follows (where $\varphi_{S}$ is a predicate over $S$, $e$ is label of $L$ and $I$ is a time interval) :

$$
f::=\varphi_{S}|e| \dot{\neg} f|f \dot{\wedge} f| f \mathcal{U}_{I} f
$$

Remarks. As usual, we define the three temporal operators $\diamond$ (eventually), $\square$ (always) and $\mathcal{W}$ (weak until) as $\diamond_{I} f \stackrel{\text { def }}{=} \top \mathcal{U}_{I} f, \square_{I} f \stackrel{\text { def }}{=} \neg\left(\top \mathcal{U}_{I} \neg f\right)$ and $f_{1} \mathcal{W}_{I} f_{2} \stackrel{\text { def }}{=}\left(f_{1} \mathcal{U}_{I} f_{2}\right) \dot{\vee}\left(\square_{\overleftarrow{I}} f_{1}\right)$, where $\mathcal{U}_{I}$ is the until operator constrained with a time interval. We introduce as well the connectors $\Rightarrow$ and $\dot{\vee}$. The semantics of this logic is defined over our formalization of runs and its implementation is available in our CoQ framework.

Then, in order to reason and prove the correctness of real-time specifications modelled as TCTSs, we have embedded in our CoQ framework a proof methodology very close to the one proposed in [15], based on the same class of real-time properties they consider (bounded response, bounded unless and bounded invariance properties) expressed as timed-SELTL formulæ. To do so, we have established several COQ theorems such as the two given below, where $p, q, r$ and $\Phi$ are predicates over $S$. These theorems provide efficient proof rules since they enable us to certify in CoQ real-time specifications expressed as timed-SELTL formulæ over runs (their conclusion), but reasoning locally (thanks to their premises) on TCTS $s$ with Hoare triple and logical formulae. We have also proved transitivity and disjunction lemmas which are essential to make the method usable.

4. Carlos D.Luna has defined in CoQ the TCTL tree logic : http://coq.inria.fr/ pylons/contribs/view/CTLTCTL/v8.4 


$$
\begin{aligned}
& \text { (1) } \forall s, p(s) \Rightarrow \neg \boldsymbol{e n b}_{s}(t r) \\
& \text { (2) } \forall s, p(s) \Rightarrow \Phi(s) \\
& \text { (3) } \forall t r^{\prime} \neq t r,\{\Phi\} t r^{\prime}\{\Phi\} \\
& \text { (4) } \forall s, \Phi(s) \Rightarrow q(s) \\
& \frac{(5) \forall s, \Phi(s) \wedge \boldsymbol{e n b}_{s}(t r) \Rightarrow r(s)}{\forall \sigma, \forall i, \sigma \models_{i} p \dot{\Rightarrow} q \mathcal{W}_{\vec{I}_{t r}} r}
\end{aligned}
$$

$$
\begin{aligned}
& \text { (1) } \forall s, p(s) \Rightarrow \Phi(s) \vee q(s) \\
& \text { (2) } \forall s, \Phi(s) \Rightarrow \text { enb }_{s}(t r) \\
& \text { (3) } \forall s, \forall t r^{\prime} \neq t r, \Phi(s) \wedge \text { enb }_{s}\left(t r^{\prime}\right) \\
& \Rightarrow \min \left(I_{t r^{\prime}}\right)>0 \wedge\left(t r^{\prime}, t r\right) \notin \mathcal{R} \\
& \text { (4) } \forall t r^{\prime} \neq t r,\{\Phi\} t r^{\prime}\{\Phi \vee q\} \\
& \frac{(5)\{\Phi\} \operatorname{tr}\{q\} \wedge \max \left(I_{t r}\right)<\infty}{\forall \sigma, \forall i, \sigma \models_{i} p \Rightarrow \diamond_{I_{t r}} q}
\end{aligned}
$$

\section{Conclusions}

We have presented a mathematical model for specifying and reasoning over realtime systems. This model enables us to specify some very subtle timed-semantic differences of fine-grained constructs (see Section 1.1) allowed by modern realtime component-based languages such as FIACRE. Also, we provide logical and functional definitions to give semantic interpretations of real-time systems specified as TCTS $s$ in terms of well known formalisms that allow to reason over their timing and temporal aspects. All definitions and theorems presented in this paper have been fully formalized and established in the CoQ proof assistant ${ }^{5}$.

We stress also in this conclusion that we do not address the whole FIACRE language [6] semantics in this paper. However, we consider the introduced model of TCTS (together with [12]) as the semantic kernel of FIACRE and the cornerstone of future research and developments. Transition systems with real-time constraints and priorities are very complicated and reasoning about them is tedious. But on the other hand such systems are widely used in real-life embedded systems, especially in spatial and avionics domains, which is a good motivator for developing a fully mechanized framework. This makes the presented work a very useful starting point for further theoretical or applied developments.

Future work will consist in certifying through the use of CoQ some existing patterns used in the design of avionic embedded applications, such as the periodic controller presented in [8]. Also, an application of this work is to certify within COQ the translation of timed temporal formulæ into FIACRE observers [1]. Another envisioned application of our semantic framework concerns the verification of transformations between timed formalisms such as the one presented in [5], or model simplification, as for example, the flattening operator of component-based languages. At last, we are convinced that this work together with [12] could be a good basis for the certified compilation [19] of the FIACRE language.

\section{References}

1. N. Abid, S. D. Zilio, and D. L. Botlan. A Verified Approach for Checking Real-Time Specification Patterns. In Proceedings of VeCos, 2012.

5. available at http://www.irit.fr/ Manuel. Garnacho/Coq/FORMATS13. 
2. A. Arnold. Finite Transition Systems - Semantics of Communicating Systems. Prentice Hall international series in computer science. Prentice Hall, 1994.

3. A. Basu, M. Bozga, and J. Sifakis. Modeling Heterogeneous Real-time Components in BIP. In SEFM, pages 3-12, 2006.

4. B. Bérard, F. Cassez, S. Haddad, D. Lime, and O. H. Roux. Comparison of Different Semantics for Time Petri Nets. In ATVA, pages 293-307, 2005.

5. B. Bérard, F. Cassez, S. Haddad, D. Lime, and O. H. Roux. Comparison of the Expressiveness of Timed Automata and Time Petri Nets. In FORMATS, pages 211-225, 2005.

6. B. Berthomieu, J.-P. Bodeveix, P. Farail, M. Filali, H. Garavel, P. Gaufillet, F. Lang, and F. Vernadat. Fiacre: an Intermediate Language for Model Verification in the Topcased Environment. In ERTS 2008, 2008.

7. Y. Bertot and P. Castéran. Interactive Theorem Proving and Program Development (Coq'Art: The Calculus of Inductive Constructions). Texts in Theoretical Computer Science. Springer, 2004.

8. J.-P. Bodeveix, M. Filali, M. Garnacho, R. Spadotti, and Z. Yang. On the Mechanization of an AADL Subset. Science of Computer Programming : special issue on Architecture Design Language, 2013. Submitted.

9. S. Chaki, E. M. Clarke, J. Ouaknine, N. Sharygina, and N. Sinha. State/EventBased Software Model Checking. In IFM, pages 128-147, 2004.

10. E. A. Emerson and J. Y. Halpern. Decision Procedures and Expressiveness in the Temporal Logic of Branching Time. In STOC, pages 169-180, 1982.

11. The FIACRE Specification Language for Real-Time Concurrent Systems. http://projects.laas.fr/fiacre/.

12. M. Garnacho, J.-P. Bodeveix, and M. Filali. Mechanized Semantics of Concurrent Systems with Priorities. IRIT Research Report-2013-16-FR, 2013. http://www . irit.fr/ Manuel.Garnacho/Publications/MechPrio.pdf.

13. H. Geuvers, A. Koprowski, D. Synek, and E. van der Weegen. Automated MachineChecked Hybrid System Safety Proofs. In ITP, pages 259-274, 2010.

14. R. Hale, R. Cardell-Oliver, and J. Herbert. An Embedding of Timed Transition Systems in HOL. FMSD, 3(1/2):151-174, 1993.

15. T. A. Henzinger, Z. Manna, and A. Pnueli. Temporal Proof Methodologies for Real-time Systems. In POPL, pages 353-366, 1991.

16. T. A. Henzinger, Z. Manna, and A. Pnueli. Timed Transition Systems. In REX Workshop, pages 226-251, 1991.

17. C. A. R. Hoare. Communicating Sequential Processes. Prentice-Hall, 1985.

18. The ISABELLE System. http://isabelle.in.tum.de/.

19. X. Leroy. Formal certification of a compiler back-end, or: programming a compiler with a proof assistant. In 33rd symposium Principles of Programming Languages, pages 42-54. ACM Press, 2006.

20. C. Paulin-Mohring. Modelisation of Timed Automata in Coq. In TACS, pages 298-315, 2001.

21. A. Pnueli. The Temporal Logic of Programs. In FOCS, pages 46-57, 1977.

22. J. Rushby. Mechanized Formal Methods: Progress and Prospects. In Proc. of FSTTCS, volume 1180 of LNCS, pages 43-51, Hyderabad, India, 1996. 